\title{
Coronary Plaque Geometry and Thoracic Fat Distribution in Patients with Acute Chest Pain - a CT Angiography Study
}

\author{
Mihaela Ratiu ${ }^{1}$, Nora Rat ${ }^{1,2}$, Tiberiu Nyulas ${ }^{1,2}$, Geanina Moldovan ${ }^{1}$, Victoria Rus ${ }^{1}$, \\ Theodora Benedek ${ }^{1,2}$, Imre Benedek ${ }^{1,2}$ \\ ${ }^{1}$ University of Medicine, Pharmacy, Sciences and Technology, Tîrgu Mureș, Romania \\ ${ }^{2}$ Department of Computational Imaging, Cardio Med Medical Center, Tîrgu Mureș, Romania
}

\section{ABSTRACT}

The aim of our study was to investigate the correlation between volumes of thoracic fat distributed in different compartments and the geometry of vulnerable coronary plaques assessed by coronary computed tomography angiography (CCTA), in patients with acute chest pain. Methods: This was a non-randomized, observational, single-center study, including 50 patients who presented in the emergency department with acute chest pain who underwent 128-slice single-source CCTA. Plaque geometry was evaluated in transversal and longitudinal planes, and the assessment of adipose tissue was performed using the Syngo.via Frontier (Siemens AG, Healthcare Sector, Forchheim, Germany) research platform. Results: Eccentric plaques presented a significantly higher incidence of spotty calcification ( $40 \% \mathrm{vs} .22 \%$, $\mathrm{p}=0.018$ ), whereas positive remodeling, volume of low attenuation plaque, and incidence of napkin-ring sign were not significantly different between the study groups or in ascending versus descending plaques. The volume of pericoronary fat around the plaque was significantly larger near eccentric lesions $\left(707.68 \pm 454.08 \mathrm{~mm}^{3}\right.$ vs. $\left.483.25 \pm 306.98 \mathrm{~mm}^{3}, \mathrm{p}=0.046\right)$ and descendent plaques $\left(778.26 \pm 479.37 \mathrm{~mm}^{3}\right.$ vs. $\left.473.60 \pm 285.27 \mathrm{~mm}^{3}, \mathrm{p}=0.016\right)$. Compared to ascending lesions, descendent ones presented a significantly larger volume of thoracic fat $(1,599.25 \pm 589.12 \mathrm{~mL}$ vs. $1,240.71 \pm 291.50 \mathrm{~mL})$, while there was no significant correlation between thoracic fat and cross-sectional eccentricity. Conclusions: The phenotype of plaque distribution and geometry seems to be associated with a higher vulnerability of coronary lesions and may be influenced by the local accumulation of inflammatory mediators released by the pericoronary epicardial adipose tissue.

Keywords: pericoronary fat, plaque geometry, CCTA, acute chest pain, vulnerable coronary lesions

\section{ARTICLE HISTORY}

Received: August 15, 2018

Accepted: December 21, 2018

\section{CORRESPONDENCE}

Tiberiu Nyulas

Str. 22 Decembrie 1989 nr. 76

540124 Tîrgu Mureș, Romania

Tel: +40 265217333

E-mail: tiberiu.nyulas@gmail.com

\footnotetext{
Mihaela Ratiu: Str. Gheorghe Marinescu nr. 38, 540139 Tîrgu Mureș, Romania. Tel: +40 265 215 551, E-mail: d_a_mihaela@yahoo.com Nora Rat: Str. 22 Decembrie 1989 nr. 76, 540124 Tîrgu Mureș, Romania. Tel: +40 265217 333, E-mail: ratnora@gmail.com Geanina Moldovan: Str. Gheorghe Marinescu nr. 38, 540139 Tîrgu Mureș, Romania. Tel: +40 265 215 551, E-mail: geanina78moldovan@yahoo.com Victoria Rus: Str. Gheorghe Marinescu nr. 38, 540139 Tîrgu Mureș, Romania. Tel: +40 265215 551, E-mail: victoriarus91@yahoo.com Theodora Benedek: Str. Gheorghe Marinescu nr. 38, 540139 Tîrgu Mureș, Romania. Tel: +40 265 215 551, E-mail: theodora.benedek@gmail.com Imre Benedek: Str. Gheorghe Marinescu nr. 38, 540139 Tîrgu Mureș, Romania. Tel: +40 265 215 551, E-mail: imrebenedek@yahoo.com
} 


\section{BACKGROUND}

Coronary computed tomography angiography (CCTA) has emerged as a valuable imaging tool to evaluate plaque morphology and composition in patients with coronary artery disease. Moreover, CCTA can detect particular features of atheromatous plaques which have been correlated with an increased risk of an acute coronary event such as spotty calcifications, positive remodeling, low attenuation, and napkin-ring sign., ${ }^{1,2}$

However, very few studies have addressed so far the issue of lesion geometry, which can play a significant role in the complex process of coronary plaque vulnerabilization. Especially plaque eccentricity (PE), a morphological feature reflecting a distinct pattern of atheroma geometry, may influence the coronary wall shear stress acting at the level of luminal narrowing. Since recent data demonstrated the role of coronary wall shear stress in plaque vulnerabilization, PE could represent another morphological feature of atheromatous plaque vulnerability. A recent intravascular ultrasound study demonstrated that culprit lesions are usually eccentric, while a combined intravascular ultrasound and optical coherence tomography study reported that lesions with signs of erosion presented a higher eccentricity index. ${ }^{3,4}$

PE can be assessed on two different planes: (1) the transverse plane, classifying lesions into concentric or eccentric ones, and (2) the longitudinal plane, classifying lesions into ascending or descending ones.

However, coronary plaque vulnerabilization is a more complex process influenced not only by local hemorrheologic factors but also by the level of local and systemic inflammation. One of the main sources of inflammatory factors acting at the level of coronary arteries is represented by thoracic fat, which can serve as an inflammation mediator. Although the relation between epicardial fat volume and coronary plaque morphology has been well documented, the correlation between plaque morphology and fat depots at the level of different thoracic compartments has not been investigated so far.

Adipose tissue, present at different levels in the thorax, has multifactorial roles in cardiovascular diseases, being distributed in the subcutaneous or visceral compartment, which includes epicardial fat and extrapericardial fat. As a fragment of epicardial fat, pericoronary adipose tissue, located in the immediate neighborhood of a coronary segment, can be also identified and quantified.

Fat deposits around the heart were identified as key factors associated with a higher incidence and severity of coronary artery stenosis. ${ }^{5}$ Several studies suggest that a larger volume of epicardial fat is associated with an increased risk of myocardial ischemia or acute coronary syndromes. ${ }^{6,7}$ Furthermore, a recent hypothesis suggests that epicardial adipose tissue represents a source of inflammatory mediators exerting a direct local effect on the coronary arteries and leading to significant changes in atheromatous plaque architecture at this level. ${ }^{8}$

The aim of our study was to investigate the correlation between volumes of thoracic fat distributed in different compartments and the geometry of vulnerable coronary plaques assessed by CCTA, in patients with acute chest pain.

\section{MATERIAL AND METHODS}

\section{STUDY POPULATION}

This was a non-randomized, observational, single-center study, including 50 patients who presented in the emergency department with acute chest pain. In all patients, the electrocardiogram did not reveal any ST-segment elevation, and troponin values were inconclusive. Therefore, all subjects underwent CCTA for the assessment of coronary circulation. Only subjects with at least one significant stenosis with vulnerability features were enrolled in the study. The exclusion criteria included renal failure, chronic kidney disease, pregnancy, lactation, allergy to iodine, and active malignancy.

\section{CCTA ACQUISITION AND IMAGE PROCESSING}

CCTA was performed with a 128-slice single-source CT scanner (Somatom Definition AS, Siemens AG, Healthcare Sector, Forchheim, Germany) with retrospective electrocardiographic gating. Scanning parameters were: tube voltage $100 \mathrm{kv}$, tube current $180 \mathrm{mAs} /$ rotation, gantry rotation time $330 \mathrm{msec}$, pitch adapted to heart rate, collimation $128 \times 0.6$. The contrast agent was injected with a flow rate of $5 \mathrm{~mL} / \mathrm{s}$ in the antecubital vein, using an 18-gauge venous catheter placed in the right cubital fossa. Contrast quantity was adapted to patient body weight and was followed by saline flush. The region of interest was placed in the descending aorta, and the threshold was $180 \mathrm{HU}$. Datasets were retrospectively reconstructed with a slice thickness of 0.6 using medium soft-tissue convolution kernel, and the best image quality phase was selected for post processing. Images were further evaluated semi-automatically using a 3D contour detection algorithm provided by Syngo.via Multimodality Workplace. 


\section{PLAQUE MORPHOLOGY AND GEOMETRY}

The assessment of coronary plaque morphology was performed using a semi-automatic software with 3D reconstructions and included quantification of stenosis degree, luminal volume, plaque volume, and density-based plaque composition (calcified, fibrotic, or lipid-rich atheroma). Vulnerable features evaluated included: low attenuation plaque (defined as density $<50 \mathrm{HU}$ in $\geq 10 \%$ of the plaque), positive remodeling (defined by a remodeling index $\geq 1.1$ ), spotty calcification (identified as areas with dimensions $<3 \mathrm{~mm}$ and density $\geq 130 \mathrm{HU}$ ), and napkin-ring sign.

Plaque geometry was evaluated in two directions: transversal and longitudinal.

Cross-sectional plaque eccentricity (CE) was assessed in a transverse section at the level of maximum stenosis. The CE index was calculated as: (maximum wall thickness - minimum wall thickness) divided by maximum wall thickness. Concentric lesions were defined as having a CE $<0.3$, whereas eccentric lesions were defined as having a $\mathrm{CE} \geq 0.3$.

Longitudinal plaque eccentricity (LE) referred to stenosis with abrupt narrowing on the proximal or distal edge and was assessed according to the location of maximum stenosis in a longitudinal section. The LE index was calculated as the distance between the proximal end of the lesion and the place of maximum stenosis divided by lesion length. Descendent lesions were defined by $L E<0.5$, whereas ascendant lesions were defined by a $\mathrm{LE} \geq 0.5$.

\section{ASSESSMENT OF THORACIC FAT COMPARTMENTS}

The evaluation of adipose tissue was performed using the research platform Syngo.via Frontier (Siemens AG, Healthcare Sector, Forchheim, Germany) and a dedicated software for cardiovascular risk assessment, which marks the adipose tissue automatically based on its specific density, calculating the volumes of epicardial fat, as well as visceral and subcutaneous thoracic fat. The evaluation of pericoronary adipose tissue was performed manually, in the segment adjacent to the culprit coronary stenosis by marking $1 \mathrm{~cm}$ around the lesion, or by marking the nearest anatomical structure with different density if this was closer than $1 \mathrm{~cm}$.

\section{STUDY GROUPS}

The study lot was divided according to CE into two groups: 25 (50\%) patients with concentric culprit lesions and 25 $(50 \%)$ patients with eccentric culprit lesions. According to
LE, $20(40 \%)$ of the culprit lesions were descendent and $30(60 \%)$ of them were ascendant.

\section{STATISTICAL ANALYSIS}

All data were analyzed using Graph Pad 3.10 software (GraphPad Software, Inc., San Diego, USA). All data processed were checked for normality. Categorical variables were expressed in numbers and percentage and were compared using the Fisher test. Continuous variables were presented as mean \pm standard deviation and were analyzed using the unpaired Student's t-test for normally distributed variables and the Mann-Whitney test for nonnormally distributed variables. The threshold for statistical significance was set at 0.05 .

\section{ETHICAL CONSIDERATIONS}

All study procedures were conducted in compliance with the Declaration of Helsinki, and all patients signed an informed consent prior to enrollment in the study.

\section{RESULTS}

\section{BASELINE CHARACTERISTICS OF THE STUDY POPULATION}

The average age of the patients included in the study was $60.36 \pm 11.71$ years, and most of them were males (38, $76.0 \%$ ). Regarding gender, obesity, hypertension, dyslipidemia, smoking status, left ventricular ejection fraction, calcium score, history of coronary artery disease, myocardial infarction, or stroke, there were no significant differences between the two groups. However, patients with eccentric plaques had more frequently diabetes ( $5 \%$ vs. $14 \%$, $\mathrm{p}=0.018)$. The characteristics of the study population are presented in Table 1.

Patients with ascendant lesions were more frequently males and obese compared to patients with descendant lesions ( $24 \%$ vs. $52 \%, p=0.044$ and $5 \%$ vs. $48 \%, p=$ 0.034, respectively).

\section{PLAQUE GEOMETRY AND CORONARY PLAQUES CCTA FEATURES}

There were no significant differences between the study groups with concentric versus eccentric lesions regarding lesion location, length, diameter stenosis, minimal luminal area, and vessel and luminal volumes. This was also the case in the sub-analysis of ascendant versus descendent 
TABLE 1. Clinical characteristics of the study population

\begin{tabular}{|c|c|c|c|c|c|c|c|}
\hline & All $(n=50)$ & $\begin{array}{c}\text { Concentric } \\
\text { lesions } \\
\text { EI }<0.3 \\
(\mathbf{n}=25)\end{array}$ & $\begin{array}{c}\text { Eccentric } \\
\text { lesions } \\
\text { EI } \geq 0.3 \\
(\mathbf{n}=25)\end{array}$ & $p$ value & $\begin{array}{c}\text { Descendent } \\
\text { lesions } \\
\text { LEI <0.5 } \\
(\mathbf{n}=\mathbf{2 0})\end{array}$ & $\begin{array}{c}\text { Ascendant } \\
\text { lesions } \\
\text { LEI } \geq 0.5 \\
(\mathrm{n}=30)\end{array}$ & p value \\
\hline Age, years & $60.36 \pm 11.71$ & $61 \pm 11.32$ & $59.72 \pm 12.28$ & 0.703 & $59.7 \pm 12.82$ & $60.8 \pm 11.11$ & 0.756 \\
\hline Gender, male, n (\%) & $38(76)$ & $19(38)$ & $19(38)$ & 1.258 & $12(24)$ & $26(52)$ & 0.044 \\
\hline Obesity, BMI $>25 \mathrm{~kg} / \mathrm{m}^{2}, \mathrm{n}(\%)$ & $34(68)$ & $14(28)$ & $20(40)$ & 0.128 & $10(5)$ & $24(48)$ & 0.034 \\
\hline Hypertension, n (\%) & $38(76)$ & $18(36)$ & $20(40)$ & 0.741 & $15(30)$ & $23(46)$ & 1.000 \\
\hline Dyslipidemia, n (\%) & $30(60)$ & $15(30)$ & $15(30)$ & 1.226 & $9(18)$ & $21(42)$ & 0.088 \\
\hline Diabetes, $\mathrm{n}(\%)$ & $19(38)$ & $5(10)$ & $14(28)$ & 0.018 & $8(16)$ & $11(22)$ & 1.000 \\
\hline Current smoking, n (\%) & $27(54)$ & $12(24)$ & $15(30)$ & 0.570 & $10(5)$ & $17(34)$ & 0.773 \\
\hline Previous $\mathrm{CAD}, \mathrm{n}(\%)$ & $21(42)$ & $8(16)$ & $13(26)$ & 0.251 & $8(16)$ & $13(26)$ & 1.000 \\
\hline Previous myocardial infarction $\mathrm{n}(\%)$ & $13(26)$ & $5(10)$ & $8(16)$ & 0.520 & $4(8)$ & $9(18)$ & 0.521 \\
\hline Previous stroke, n (\%) & $7(14)$ & $4(8)$ & $3(6)$ & 1.000 & $2(4)$ & $5(10)$ & 0.687 \\
\hline Ca score, mean \pm SD & $170.7 \pm 208.41$ & $194.52 \pm 197.71$ & $146.88 \pm 220.02$ & 0.168 & $\begin{array}{r}164.75 \pm 207.80 \\
109.00-765.00\end{array}$ & $\begin{array}{c}174.66 \pm 212.27 \\
83.50-813.00\end{array}$ & 0.781 \\
\hline $95 \%$ confidence interval & $111.41-229.99$ & $112.91-276.13$ & & & $109.00-765.00$ & $83.50-813.00$ & \\
\hline $\begin{array}{l}\text { LVEF }(\%) \text {, mean } \pm \text { SD } \\
95 \% \text { confidence interval }\end{array}$ & $1.22(0.3-8.2)$ & & & 0.668 & $\begin{array}{l}49.00 \pm 2.95 \\
48.50-55.00\end{array}$ & $\begin{array}{l}48.56 \pm 5.39 \\
49.00-60.00\end{array}$ & 0.838 \\
\hline
\end{tabular}

TABLE 2. CCTA features of the culprit lesions

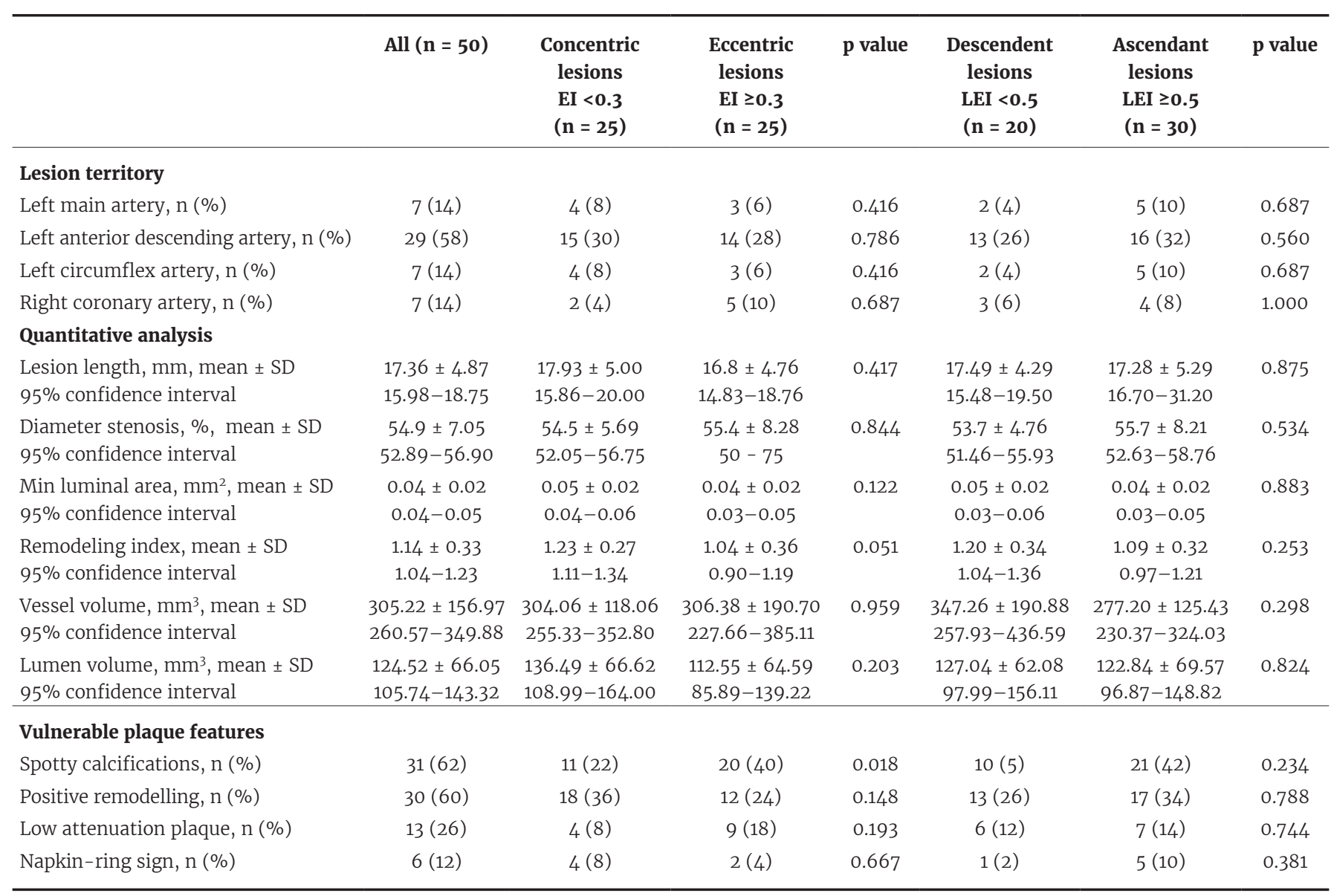

plaques. However, eccentric plaques presented a lower remodeling index compared with concentric ones $(1.04 \pm 0.36$ vs. $1.23 \pm 0.27, p=0.051)$. In respect to vulnerability features of the coronary plaques, eccentric plaques presented a significantly higher frequency of spotty calcification ( $40 \%$ vs. $22 \%, p=0.018)$, whereas positive remodeling, low attenu- 

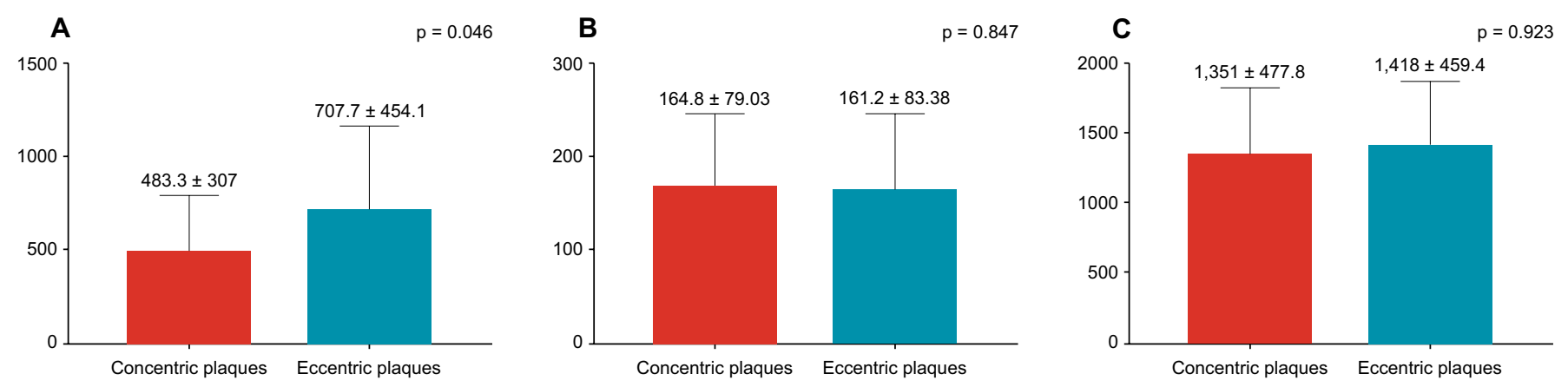

FIGURE 1. The association between cross-sectional plaque eccentricity and the volume of thoracic fat in different compartments. A Pericoronary fat around plaque $(\mathrm{mm} 3)$; B - Epicardial fat $(\mathrm{mL}) ; \mathbf{C}-$ Thoracic fat $(\mathrm{mL})$
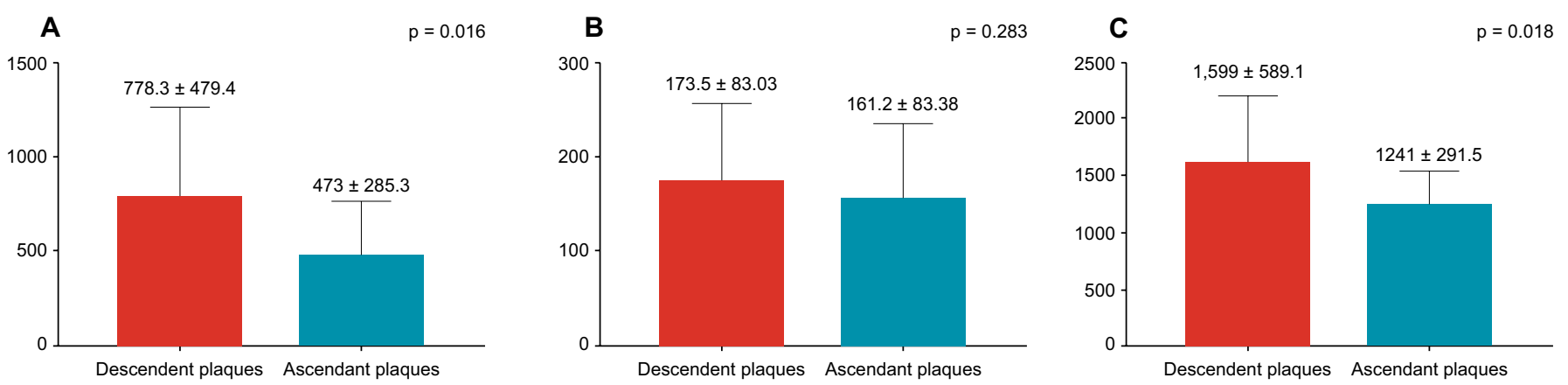

FIGURE 1. The association between longitudinal plaque eccentricity and the volume of thoracic fat in different compartments. A - Pericoronary fat around plaque (mm3); B - Epicardial fat $(\mathrm{mL}) ; \mathbf{C}-$ Thoracic fat $(\mathrm{mL})$

ation plaque, and napkin-ring sign were not significantly different between the study groups or in ascending versus descending plaques. CCTA features of coronary plaques in the study groups are presented in Table 2.

\section{PLAQUE GEOMETRY AND ADIPOSE TISSUE DISTRIBUTION}

The CCTA analysis of plaque eccentricity and CCTA quantification of thoracic fat in different compartments revealed that pericoronary fat around the plaque was present in a significantly larger volume near eccentric lesions (707.68 $\pm 454.08 \mathrm{~mm}^{3}$ vs. $\left.483.25 \pm 306.98 \mathrm{~mm}^{3}, \mathrm{p}=0.046\right)$ and descendent plaques $\left(778.26 \pm 479.37 \mathrm{~mm}^{3}\right.$ vs. $473.60 \pm 285.27$ $\mathrm{mm}^{3}, \mathrm{p}=0.016$ ), while there was no significant correlation between the amount of thoracic adipose tissue and crosssectional eccentricity. The volume of epicardial fat was not significantly different in the two study groups or in patients with ascendant versus descendent lesions (Figures 1 and 2).

\section{DISCUSSIONS}

The present study aimed to investigate the correlation between fat distribution in different thoracic compartments and the morphological features of high-risk coronary plaques. CCTA is nowadays a useful tool used for a complex evaluation of coronary artery disease. This is not only because of its noninvasive character but also of the multiple data sets that can be obtained beside the assessment of coronary circulation.

The evaluation of adipose tissue surrounding the heart has multifactorial implications in cardiovascular diseases and is a highly investigated topic. Modern CCTA scanners and post-processing softwares allow the computation of adipose tissue due to its specific negative Hounsfield units. Several studies demonstrated the correlation between adipose tissue and an overall increased cardiovascular risk, and an increased frequency of high risk coronary plaques. ${ }^{9,10}$ Another study showed that the volumes of epicardial fat are significantly higher in patients with acute coronary syndromes compared to stable subjects, identifying also more vulnerable plaques in patients with larger epicardial adipose depots. ${ }^{11}$

Another study which evaluated local fat around coronary plaques proved that pericoronary adipose tissue is a more reliable marker of plaque vulnerability than global epicardial fat or total intrathoracic fat. ${ }^{12}$

Due to its local paracrine effect, pericoronary plaque adipose tissue can trigger not only atheroma formation but 
also plaque progression, vulnerabilization, and rupture. ${ }^{13}$ Patients with type 2 diabetes mellitus and acute myocardial infarction show usually a direct correlation between epicardial adipose tissue thickness and the persistence of marked inflammation in the post-infarction period, and poorer outcomes reflected by lower ejection fraction, ventricular enlargement, and remodeling at six months.

On the other hand, coronary plaque geometry is still an underinvestigated subject. A recent FFR-based study demonstrated that coronary geometry has a direct impact on the functional significance of a coronary stenosis. ${ }^{14}$ Postmortem studies revealed that plaque rupture occurs more often in eccentric plaques. ${ }^{15} \mathrm{~A}$ series of studies have been recently published in the literature which show a complex relation between plaque geometry, hemodynamic forces, and atheroma vulnerability. ${ }^{16}$

Our study found a significant correlation between pericoronary fat volumes and eccentric lesions in the transversal section, as well as between pericoronary fat and descendant lesions. This finding may suggest that specific geometrical phenotypes can be associated with a higher vulnerability of atheromatous coronary plaques and with a specific pattern of epicardial fat distribution in the vicinity of the lesion.

\section{CONCLUSIONS}

Coronary plaques which exhibit an increased eccentricity, such as cross-sectional eccentric and descendant ones, are associated with a significantly larger volume of pericoronary fat around the atheromatous plaques but not of the total epicardial adipose tissue, while descendant lesions are associated with a larger total thoracic fat volume. The phenotype of plaque distribution and geometry seems to be associated with a higher vulnerability of the coronary lesions and may be influenced by the local accumulation of inflammatory mediators released by the pericoronary epicardial adipose tissue.

\section{CONFLICT OF INTEREST}

Nothing to declare.

\section{ACKNOWLEDGEMENT}

The study is part of the PhD study entitled "Three dimensional imaging methods used in high risk plaques characterization", financed by the program of doctoral research of the University of Medicine and Pharmacy of Tîrgu Mureș, Romania, contract number 13406/17 from 07.10.2015.

\section{REFERENCES}

1. Pozo E, Agudo-Quilez P, Rojas-Gonzales A, et al. Noninvasive diagnosis of vulnerable coronary plaque. World J Cardiol. 2016;8:520-533. doi: 10.4330/wjc.v8.i9.520.

2. Benedek T, Gyongyosi M, Benedek I. Multislice computed tomographic coronary angiography for quantitative assessment of culprit lesions in acute coronary syndromes. Can J Cardiol. 2013;29:364-371. doi: 10.1016/j.cjca.2012.11.004.

3. Yamagishi M, Terashima M, Awano K, et al. Morphology of vulnerable coronary plaque: insights from follow-up of patients examined by intravscular ultrasound before an acute coronary syndrome. J Am Coll Cardiol. 2000;35:106-111.

4. Higuma $\mathrm{T}$, Soeda $\mathrm{T}$, Abe $\mathrm{N}$, et al. A Combined Optical Coherence Tomography and Intravascular Ultrasound Study on Plaque Rupture, Plaque Erosion, and Calcified Nodule in Patinets With ST-Segment Elevation Myocardial Infarction: Incidence, Morphologic Characteristics, and Outcomes After Percutaneous Coronary Intervention. JACC Cardiovasc Interv. 2015;8:1166-1176. doi: 10.1016/j.jcin.2015.02.026

5. Hedgire S, Baliyan V, Zucker EJ, et al. Perivascular Epicardial Fat Stranding at Coronary CT Angiography: A Marker of Acute Plaque Rupture and Spontaneous Coronary Artery Dissection. Radiology. 2018;287:808-815. doi: 10.1148/radiol.2017171568.

6. Harada K, Amano T, Uetani T, et al. Cardiac 64-multislice computed tomography reveals increased epicardial fat volume in patients with acute coronary syndrome. Am J Cardiol. 2011;108:1119-1123. doi: 10.1016/j.amjcard.2011.06.012.

7. Tamarappoo B, Dey D, Shmilovich $\mathrm{H}$, et al. Increased pericardial fat volume measured from noncontrast CT predicts myocardial ischemia by SPECT. JACC Cardiovasc Imaging. 2010;3:1104-1112. doi: 10.1016/j.jcmg.2010.07.014.

8. MazurekT, Zhang L, Zalewski A, et al. Human epicardial adipose tissue is a source of inflammatory mediators. Circulation. 2003;108:2460-2466. doi: 10.1161/01.CIR.0000099542.57313. C5.

9. Mahabadi AA, Massaro JM, Rosito GA, et al. Association of pericardial fat, intrathoracic fat, and visceral abdominal fat with cardiovascular disease burden: the Framingham Heart Study. Eur Heart J. 2009;30:850-856. doi: 10.1093/eurheartj/ ehn573.

10. Ding J, Hsu FC, Harris TB, et al. The association of pericardial fat with incident coronary heart disease: the Multi-Ethnic Study of Atherosclerosis (MESA). Am J Clin Nutr. 2009;90:499504. doi: 10.3945/ajcn.2008.27358.

11. Nyulas T, Morariu M, Rat N, et al. Epicardial Adipose Tissue Role as a Marker of Higher Vulnerability in Patients with Coronary Artery Disease. Journal of Interdisciplinary Medicine. 2018;3:77-83. doi: 10.2478/jim-2018-0018.

12. Rat N, Opincariu D, Blendea C, et al. The Effect of Periplaque Fat on Coronary Plaque Vulnerability in Patients with Stabile Coronary Artery Disease - a 128-slice CT-based Study. Journal of Interdisciplinary Medicine. 2018;3:67-76. doi: 10.2478/jim2018-0019.

13. Maurovich-Horvath P, Kallianos K, Engel LC, et al. Influence of pericoronary adipose tissue on local coronary atherosclerosis as assessed by a novel MDCT volumetric method. Atherosclerosis. 2011;219:151-157. doi: 10.1016/j. atherosclerosis.2011.06.049. 
14. Kang DY, Ahn JM, Kim YK, et al. Impact of Coronary Lesion Geometry on fractional Flow Reserve: Data From International Cardiology Research In-Cooperation Society-Fractional Flow Reserve and Intravascular Ultrasound Registry. Circ Cardiovasc Imaging. 2018;11:e007087. doi: 10.1161/ CIRCIMAGING.117.007087.

15. Jain S, Biligi D. An Autopsy Study on Coronary Atherosclerosis with Morphological and Morphometric Analysis. Int J Sci Res. 2013;4:1522-1526.

16. Puri R, Leong DP, Nicholls SJ, et al. Coronary artery wall shear stress is associated with endothelial dysfunction and expansive arterial remodelling in patients with coronary artery disease. EuroIntervention. 2015;10:1440-1448. doi: 10.4244/EIJV10I12A249. 